\title{
INDICADORES DE QUALIDADE APLICADOS NA ASSISTÊNCIA DE ENFERMAGEM EM CUIDADOS PALIATIVOS: REVISÃO INTEGRATIVA DE LITERATURA
}

Rafaela Silva Santos Fábia Maria de Lima ${ }^{1}$ Joicy Costa da Hora ${ }^{1}$

Deuzany Bezerra de Melo Leão ${ }^{1}$ https://c

\author{
https://orcid.org/0000-0001-8541-5122 \\ https://orcid.org/0000-0001-9992-6556 \\ https://orcid.org/0000-0003-4833-7764 \\ https://orcid.org/0000-0002-5677-9135
}

Objetivo: Identificar na literatura indicadora de qualidade na assistência de enfermagem em cuidados paliativos. Método: Revisão integrativa da literatura em três bases de dados, com os descritores Nursing, palliative, indicator, palliative care, nurse care, quality indicator, cuidados paliativos, cuidado de enfermagem, tratamento paliativo, palliative assistence, indicadores de qualidade, hospice care, cuidados de conforto, atencion de enfermería, health care, indicadores de calidad, com amostra final de 7 artigos. Resultados: Dentre os artigos encontrados foi evidenciado um foco no direcionamento dos indicadores para os cuidados multiprofissional, porém, a maioria dos indicadores encontrados se aplicavam aos cuidados de enfermagem e se dividiram nos aspectos físico, psicológico, social e espiritual. Conclusão: foi encontrada uma pequena quantidade de referências nas bases de dados em relação aos indicadores em cuidados paliativos necessitando de mais estudos voltados para essa área.

Descritores: Cuidado de enfermagem; Cuidado paliativo; Indicadores de qualidade.

\section{QUALITY INDICATORS APPLIED IN NURSING CARE IN PALLIATIVE CARE: INTEGRATIVE LITERATURE REVIEW}

Objective: To identify in the literature quality indicators in nursing care in palliative care. Method: Integrative literature review in three databases with the keywords Nursing, palliative, indicator, palliative care, nurse care, quality indicator, palliative care, nursing care, palliative care, palliative assistance, quality indicators, hospice care, comfort care, nursing care, health care, quality indicators, with final sample of 7 articles. Results: Among the articles found there was a focus on the direction of indicators for multiprofessional care, however, most of the indicators found applied to nursing care and were divided into physical, psychological, social and spiritual aspects. Conclusion: A small number of references were found in the databases regarding the indicators in palliative care requiring further studies in this area.

Descriptors: Nursing care; Palliative care; Quality indicators.

\section{INDICADORES DE CALIDAD APLICADOS EN CUIDADOS DE ENFERMERÍA EN CUIDADOS PALIATIVOS: REVISIÓN INTEGRAL DE LA LITERATURA}

Objetivo: identificar en la literatura indicadores de calidad en cuidados de enfermería en cuidados paliativos. Método: Revisión integral de la literatura en tres bases de datos con las palabras clave Enfermería, paliativos, indicadores, cuidados paliativos, cuidados de enfermería, indicadores de calidad, cuidados paliativos, cuidados de enfermería, cuidados paliativos, asistencia paliativa, indicadores de calidad, cuidados paliativos, atención de confort, atención de enfermería, atención de salud, indicadores de calidad, con muestra final de 7 artículos. Resultados: Entre los artículos encontrados se enfocó la dirección de los indicadores para la atención multiprofesional, sin embargo, la mayoría de los indicadores encontrados se aplicaron a la atención de enfermería y se dividieron en aspectos físicos, psicológicos, sociales y espirituales. Conclusión: se encontró un pequeño número de referencias en las bases de datos con respecto a los indicadores en cuidados paliativos que requieren más estudios en esta área.

Descriptores: Atención de enfermeira; Cuidados paliativos; indicadores de calidad. 


\section{INTRODUÇÃO}

O desenvolvimento da tecnociência representa um importante efeito da transformação histórica na sociedade garantindo, por exemplo, a longevidade dos portadores de doenças crônicas que necessitam de cuidados a longo período. Porém, quando se trata de condições patológicas fora de possibilidade terapêutica, o uso de tratamento invasivo deve ser reconsiderado, tendo em vista a minimização do sofrimento e oferta de conforto no fim de vida ${ }^{(1)}$.

O tratamento adequado para esses problemas crônicos denomina-se cuidados paliativos, considerado uma abordagem que promove a qualidade de vida dos pacientes e seus familiares, que enfrentam doenças ameaçadoras da continuidade da vida, por meio da prevenção e alívio do sofrimento. Baseia-se em princípios que envolvem o alivio da dor e outros sintomas desagradáveis e considera a morte como um processo normal, integrando todos os aspectos sociais e psicológicos, entre outros principios que tem como principal intuito a qualidade de vida ${ }^{(2,3)}$.

Os pacientes em cuidados paliativos apresentam sintomas como fadiga, ansiedade, constipação, depressão, náusea, dor, dificuldade de concentração, distúrbio no padrão do sono entre outros. Esses problemas podem ser identificados pelo profissional de enfermagem que está na linha de frente no cuidado e em freqüente contato com esses pacientes em paliação. Além disso, enfermeiro, assim como qualquer outra profissão, necessita de busca e atualização do conhecimento, garantindo uma assistência de saúde baseada em evidências ${ }^{(4,5)}$.

Essa qualidade na prestação do serviço pode ser medida através de indicadores por ser considerado uma importante ferramenta utilizada para medir a qualidade da assistência. Esses indicadores de qualidade envolvem estrutura, processo e resultado dos cuidados prestados, baseando-se nos domínios e intervenções de enfermagem. Através deles, é possivel obter elementos de informações que são coletadas e analisadas a fim de identificar resultados sensiveis ao cuidado de enfermagem ${ }^{(3,4.6)}$.

Através desse método pode-se medir a qualidade dos cuidados paliativos, se tornando parte importante do programa, visto que, pacientes portadores de doenças graves

Quadro 1 - Estratégias de busca incuráveis e progressivas necessitam de indicação para forma de abordagem do cuidado. E com intuito de melhorar assistência desses pacientes $\mathrm{o}$ uso de indicadores voltados para assistência de enfermagem se torna crucial nesse processo ${ }^{(2.3 .6)}$

Objetivo do estudo foi identificar na literatura, indicadores de qualidade para aplicação na assistência de enfermagem em cuidados paliativos.

\section{MÉTODO}

\section{Tipo de estudo}

Trata-se de uma revisão integrativa da literatura. Essa metodologia consiste em analisar fontes secundárias de estudos a fim de sintetizar resultados nas literaturas existentes de maneira sistemática, ordenada e abrangente, contribuindo assim para as práticas de enfermagem baseado em evidência ( ${ }^{(7)}$.

\section{Procedimento metodológico}

Para elaboração desta revisão integrativa, seguem-se os seguintes estágios: identificação do problema, e a construção de uma questão norteadora: "Quais os indicadores de qualidade aplicáveis na assistência de enfermagem em cuidados paliativos presentes na literatura?", coleta de dados: análise e interpretação dos dados, organização dos dados conforme as categorias e indicadores, apresentação dos resultados e conclusões.

\section{Coleta e organização dos dados}

A coleta de dados foi realizada em abril de 2019, nas bases de dados: Literatura Latino-Americano e do Caribe em Ciências de Saúde (LILACS), Publisher Medline (PUBMED), Scientific Eletronic Library Online (SciELO), e Bases de Dados de enfermagem (BEDENF), usando os descritores e palavras chave: Nursing, palliative, indicator,palliative care, nurse care, quality indicator, cuidados paliativos, cuidado de enfermagem, tratamento paliativo, palliative assistence, indicadores de qualidade, hospice care, cuidados de conforto, atencion de enfermeria, health care, indicadores de calidad.

Foram elaboradas combinações entre os descritores e/ou palavras chave que resultaram nas seguintes estratégias de busca (Quadro 1):
LILACS e Palliative care OR Palliative assistence OR Palliative AND Nursing care OR Atencion de enfermería OR BDENF
Nursing OR Nursing indicators AND Indicators OR Quality indicators OR Indicadores de calidad. 
Palliative care OR Hospice care OR Palliative assistence OR Palliative AND Nurse OR Nursing care OR

PUBMED Atencion de enfermeria OR Nursing Indicators AND Quality indicatores OR Health care OR Indicadores de calidade OR Indicator

SCIELO

Cuidados paliativos OR Palliative care OR Assistência paliativa OR Tratamento paliativo OR Palliative AND Assistencia de enfermagem OR Nurse care OR Nursing Indicators OR Nursing AND Indicadores de enfermagem OR indicadores de qualidade OR Health care OR quality indicators OR Indicator.

\section{Análise dos dados}

A partir da coleta dos dados, foram colhidos 159 estudos, submetidos a primeira etapa de avaliação através dos seguintes critérios de inclusão e exclusão: artigos de pesquisa original, publicados nos últimos cinco anos, de forma completa em periódicos disponíveis nas bases de dados LILACS e BEDENF, PUBMED e SciELO, nos idiomas português, inglês e espanhol, de acordo com o objetivo e descritores propostos. Artigos que se encontravam em mais de uma base de dados (duplicatas), e que não atendeu aos critérios exigidos foram automaticamente excluídos. Ao final da primeira etapa de avaliação obteve um total de 122 estudos, dos quais, 86 (70,49\%) foram encontrados na PUBMED, 13 (10,65\%) na SciELO, 21 (17,21\%) na LILACS e 2 (0,16\%) na BDENF.

$\mathrm{Na}$ segunda etapa, após leitura do título e resumo dos 122 estudos, obteve um resultado de 36 artigos, sendo 27 (75\%) na PUBMED, 4 (11,11\%) na SciELO, $5(13,88)$ na LILACS e nenhum na BDENF. Ao final do processo após a terceira etapa que correspondeu a leitura na íntegra obteve-se apenas 7 estudos, sendo encontrados 4 na PUBMED, 1 na SciELO e 2 na LILACS. Os dados dessas etapas se apresentam de forma mais clara no fluxograma (figura 1).

Figura 1 - Fluxograma coleta e análise de dados.

\begin{tabular}{|c|c|c|c|}
\hline \multicolumn{4}{|c|}{$\begin{array}{l}\text { Coleta de dados } \\
\text { Total de } 159 \text { artigos encontrados }\end{array}$} \\
\hline \multicolumn{4}{|c|}{$\begin{array}{l}\text { 10 Etapa - Aplicado critérios de inclusão e exclusão } \\
\qquad 122 \text { artigos }\end{array}$} \\
\hline PUBMED & SCIELo & LILACS & BEDENF \\
\hline 86 & 13 & 21 & 2 \\
\hline \multicolumn{4}{|c|}{$\begin{array}{l}\text { 20 Etapa- Leitura do título e resumo } \\
\qquad 36 \text { artigos }\end{array}$} \\
\hline PUBMED & SCIELo & LILACS & BEDENF \\
\hline 27 & 4 & 5 & 0 \\
\hline \multicolumn{4}{|c|}{$\begin{array}{l}\text { 30 Etapa- Leitura na íntegra } \\
7 \text { artigos }\end{array}$} \\
\hline PUBMED & SCIELo & LILACS & BEDENF \\
\hline 4 & 1 & 2 & 0 \\
\hline
\end{tabular}




\section{RESULTADOS}

Os resultados foram detalhados no Tabela 1 e para facilitar a identificação foram identificados por letra com o número da referência em sobrescrito, seguidos por ano, pais, amostra e indicadores. A amostra foi para evidenciado os profissionais envolvidos nas pesquisas, enquanto que os indicadores foram para evidenciar os principais domínios e/ou indicadores encontrados, que se aplicam no cuidado de enfermagem.

Tabela 1- Características dos estudos selecionados

\begin{tabular}{|c|c|c|c|c|}
\hline № & Ano & Pais & Amostra & Indicadores aplicáveis na assistência de enfermagem \\
\hline$A^{2}$ & 2015 & EUA & $\begin{array}{l}\text { Médico, } \\
\text { enfermeiro, } \\
\text { assistente } \\
\text { social, capelão. }\end{array}$ & $\begin{array}{l}\text { Avaliação geral; dor; dispnéia; náuseas e prisão de ventre; discussão das necessidades emocional } \\
\text { ou psicológica; discussão de preocupações espirituais / religiosas; documentação de suplente; } \\
\text { preferências de tratamento e preferências de cuidado documentado; percepção da familia quanto } \\
\text { aos cuidados prestados; levantamento da familia enlutada. }\end{array}$ \\
\hline $\mathrm{B}^{5}$ & 2016 & EUA & $\begin{array}{l}\text { Médicos, } \\
\text { enfermeiros, } \\
\text { assistentes } \\
\text { sociais, } \\
\text { avaliadores e } \\
\text { usuários. }\end{array}$ & $\begin{array}{l}\text { Apoio do cuidador; localização preferida da morte; medos do paciente e da familia; negócios } \\
\text { inacabados; intervenções não farmacológicas; preferências de tratamento; capelão disponivel ou } \\
\text { um processo estabelecido para atender às necessidades espirituais; } \\
\text { qualidade das informações fornecidas; qualidade das habilidades de comunicação do provedor; } \\
\text { substituto o paciente nas decisões tomadas; documentação das decisões de cuidados; e } \\
\text { concordância entre as preferências declaradas e cuidados reais recebidos. }\end{array}$ \\
\hline
\end{tabular}

$C^{8} \quad 2014 \quad$ Indonésia $\begin{gathered}\text { Médicose } \\ \text { enfermeiros. }\end{gathered}$

Acesso a cuidados paliativos; infra-estrutura ideal com sala dedicada para discussões da equipe interdisciplinar; informações dedicadas sobre o serviço de cuidados paliativos, como folhetos ou brochuras; disponibilidade de equipamentos de enfermagem especializados, como colchão antidecúbito e fornecimento de oxigênio; disponibilidade de um instrumento validado para avaliar a dor e outros sintomas e avaliação holística das necessidades de cuidados paliativos; reunião interdisciplinar diária para discutir a gestão diária de cada paciente, bem como uma reunião semanal para rever os encaminhamentos de pacientes e planos de cuidados; ter padronizado o treinamento de indução para novos funcionários e uma avaliação anual; registro clínico estruturado para cada paciente em cuidados paliativos; um plano de tratamento multidimensional: programa de melhoria da qualidade, incluindo auditorias clínicas; registro e documentação de eventos adversos; existência de um banco de dados para registro de atividade clínica como diagnóstico, data do diagnóstico, data do encaminhamento, data de admissão aos cuidados paliativos, data e local de óbito e local de óbito preferencial.

$\begin{array}{llll}\text { Dolanda } & \text { Enfermeiras } & \begin{array}{l}\text { Serviços especializados; atendimento fora de horas; continuidade de cuidados; infra-estruturar } \\ \text { de cuidados paliativos; ferramentas de avaliação especificas; compartilhamento de informaçães, } \\ \text { documentação de dados clinicos; qualidade do atendimento e sua mensuração; educação sobre } \\ \text { cuidados paliativos. }\end{array}\end{array}$

$E^{9}$
$2018 \quad \begin{gathered}\text { Estocolmo, } \\ \text { Suécia }\end{gathered}$
Úlceras de pressão na morte; avaliação da saúde bucal na última semana de vida; alguém presente no momento da morte; informações para parentes próximos sobre a transição para os cuidados no final da vida; oferta de uma conversa de acompanhamento para o parente mais próximo depois da morte do paciente; avaliação da dor e outros sintomas durante a última semana de vida; ansiedade e náusea; e consulta de equipe especializada em cuidados paliativos durante a última semana de vida.

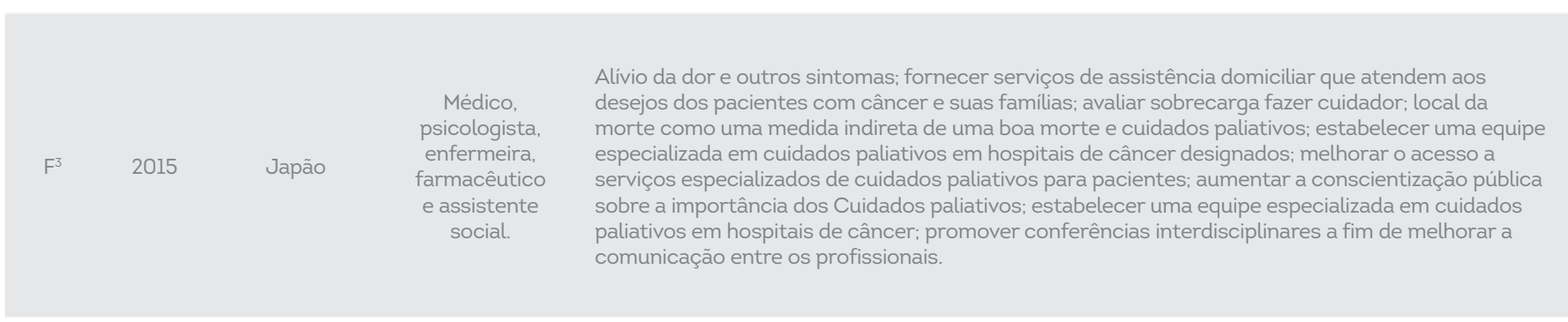

Indicador comportamental mensuração da dor na demência: carrancudo, olhos apertados, olhar tensos e olhar assustado:

olhos fechados, boca aberta, lábios apertados, olhar vazio, parecendo desinteressado, cara pálida, olhos marejados e olhar triste. 


\section{Descrição do estudo}

Os estudos analisados apresentaram uma trajetória temporal partindo do ano de 2014 a 2018. O ano de 2016 houve um maior número de publicação sendo 3 dos 7 estudos publicados no ano citado.

Em relação ao local de publicação, foram evidenciados 2 publicações nos EUA, e 5 estudos do Continente Europeu. Não foi evidenciada nenhuma publicação nacional que respondesse aos critérios e objetivo delineados nesse estudo. As amostras dos estudos encontrados envolveram, profissionais de saúde, pacientes e cuidadores, além disso, a participação o profissional de enfermagem merece destaque por está presente em todos os estudos seja na construção e/ou amostra. O foco de cuidado foi voltado para equipe multiprofissional, porém todos os estudos possuíam indicadores aplicáveis a assistência de enfermagem, presentes na Tabela 1.

\section{Indicadores do aspecto físico}

No aspecto físico, o indicador "dor" esteve presente nos estudos A, B, E, F e G, representando um importante sintoma para qualificar os cuidados prestados. Outros sintomas que foram abordados como indicador de qualidade nos aspecto físico foram a náusea presente nos estudos $A, E$ e F, a dispnéia e prisão de ventre no estudo $A$, intervenção não farmacológica nos estudos B e C. O estudo E trouxe ainda cuidados com lesões por pressão, saúde bucal e ansiedade como indicadores. Além dessa mensuração/avaliação desses sintomas, no estudo $G$, houve uma abordagem diferente. Com um foco no indicador dor, ele abrange as formas de mensuração de dor na demência, calculando esse indicador comportamental através de expressões faciais, movimentos corporais, vocalizações, ou seja, representou a forma como seria mensurado esse indicador físico da dor que inclusive foi o que mais abordado diante dos estudos.

\section{Indicadores do aspecto psicológico}

No aspecto psicológico, os estudos A e B trouxeram dois indicadores importantes que foram a discussão das necessidades emocionais e o indicador do levantamento da família enlutada. O estudo B trouxe ainda os indicadores: medo do paciente e família e negócios inacabados, trazendo a importância dessa abordagem para o bem estar psicológico do paciente/família. $\mathrm{O}$ indicador: apoio ao cuidador foi abordado nos estudos B e E, porém no C e F foi apresentada a importância de um conselheiro de luto presente, e avaliação da sobrecarga do cuidador, representando o mesmo foco do indicador anterior abordado pelos outros artigos.

\section{Indicadores do aspecto social}

Em relação aos indicadores sociais, o mais abordado nos estudos foi a documentação da preferência de tratamento presente no A, B, C, D e E. Dentre esse indicador, o estudo B ainda traz a importância de uma documentação específica sobre a localização de preferência da morte. O estudo C aborda a importância da facilidade no acesso aos cuidados paliativos como indicador social, e complementando esse indicador, o estudo F traz a assistência paliativa domiciliar como indicador importante para o comprimento de uma assistência abrangente atingindo um maior número de pessoas.

\section{Indicadores do aspecto espiritual}

$\mathrm{O}$ aspecto espiritual ainda foi pouco abordado os artigos, no qual foi destacado dois indicadores. O primeiro diz respeito a preocupações espirituais e religiosas de forma geral, indicador presente no estudo $A$, trazendo para discussão a importância da avaliação espiritual e religiosa para uma melhor assistência. Nos estudos B e C, o indicador: ter um capelão disponivel complementa também o indicador da avaliação espiritual.

Além desses, foi abordado ainda indicadores relacionados à infraestrutura e equipe multiprofissional nos estudos C, D, E e F. A presença de salas para discussão da equipe multiprofissional, passar informações sobre a importância dos cuidados paliativos para paciente e família, equipamentos de enfermagem especializados, e presença de equipe especializada nas últimas semanas de vida para planejamento de cuidados foram alguns desses indicadores.

\section{DISCUSSÃO}

A presença de publicações voltadas para esse tema trouxe a reflexão de que há uma preocupação em medir a qualidade da assistência, principalmente quando há sofrimento físico e emocional durante doenças graves e com risco de vida, considerando também que essa medição de qualidade representa parte integrante dos cuidados paliativos ${ }^{(2)}$. Essa medição de qualidade bem aferida, e com bons indicadores é necessária diante do crescimento e amadurecimento dos cuidados paliativos ${ }^{(5)}$

Nesta revisão pode-se notar, a presença freqüente, de indicadores voltados para sintomas do câncer, e apenas dois deles, estudo E e G, trazem também a doença de Alzheimer, importante por apresentar fortes sintomas assim como o câncer, e muitas vezes não tem a mesma oportunidade de acesso aos cuidados paliativos, talvez por não ser reconhecida como uma doença terminal ${ }^{(9,10)}$. Não houve um foco em indicadores específicos para determinada doença, porém todo instrumento coletado apresentou a possibilidade 
de adaptação para outra situação. Isso se deve pelo fato de que o objetivo dos indicadores seja o de auxiliar no cuidado com sintomas de difícil controle, apresentar uma mudança de visão do cuidado de um objetivo de cura para um objetivo paliativo, com intuito de melhorar as condições do paciente respeitando a subjetividade de cada um, e por tanto sendo necessária uma adaptação de cuidado, e conseqüentemente de indicadores.

Outro fator importante, evidenciado neste estudo foram a presença de indicadores de qualidade, voltados apenas para exercício de equipe multiprofissional, não havendo indicadores específicos da enfermagem, porém, no que diz respeito a participação da pesquisa, a equipe de enfermagem esteve presente em todos os estudos, pelo fato de ser uma profissão que se encontra na linha de frente do cuidado para com o paciente,e no caso de pacientes internados, oferta uma aproximação maior durante os plantões. Importante destacar também nesse ponto, a importância da abordagem multiprofissional, e em formas de cuidado que é comum a ambos profissionais que trabalham na linha de cuidados paliativos, portanto, dependendo do profissional e da peculiaridade do paciente, determinados indicadores podem ser adaptáveis a profissão, um exemplo claro diz respeito aos próprios indicadores reunidos neste estudo ${ }^{(3,6,8,9)}$

Em relação às dimensões, nos indicadores do aspecto físico, houve uma prevalência de citações no indicador: dor, e intervenções não farmacológicas para alívio de sintomas. Essa dimensão também se sobressai em relação às outras devido ao número elevado de indicadores voltados para esse aspecto. Historicamente, houve um foco para um tratamento unicamente voltado para a dimensão física, na avaliação de sinais e sintomas, e hoje ainda muito valorizada, e no que diz respeito aos cuidados paliativos deve se levar em consideração a presença de sintomas desagradáveis na terminalidade que ameaçam a qualidade de vida dos pacientes devendo ser avaliada a melhor forma de cuidar, buscando estratégias para isso como exemplo na construção de indicadores de qualidade. Porém a presença de outras dimensões como mostrado neste estudo, representam uma importância extrema para o cuidado, podendo assim interferir uma nas outras.

No aspecto psicológico os indicadores mais citados foram os relacionados a discussão das necessidades emocionais do paciente e família além do suporte para a família enlutada, quando adaptados ao cuidado de enfermagem o número de indicadores podem diminuir em relação ao aspecto físico devido a baixa abordagem relacionada a esse tema nas próprias condutas de enfermagem.

Noquedizrespeitoaosindicadoressociais, adocumentação de preferências de tratamento e local da morte apresentou uma abordagem significativa, por se tratar de uma importante forma de documentar e tornar possivel legalmente as formas de escolha de tratamento, além disso, a forma de abordagem do cuidado paliativo requer decisões difíceis como nos casos dos cuidados paliativos exclusivos, sendo necessária, por exemplo, documentos autorizando a não reanimação. Esse contato entre equipe, paciente e familia pode ser iniciado e fortificado através da equipe de enfermagem que tenha em vista a importância de se aplicar esses indicadores sociais no manejo com os cuidados paliativos.

Nos aspectos espirituais e religiosos, evidenciou uma fraca abordagem relacionada ao tema, onde apenas 3 estudos trouxeram indicadores voltados para essa dimensão. Isso retrata uma fraca abordagem e despreparo dos profissionais para lidar com questões espirituais também importantes para o bem estar físico, social e psicológico, visto que a espiritualidade também pode influenciar em outras dimensões e afetar conseqüentemente na qualidade de vida.

Alguns indicadores relacionados à infra estrutura trouxeram em sua abordagem a importância desses recursos de trabalho para uma boa assistência, desde salas especificas para atendimento de cuidados paliativos, até as formas de trabalho envolvendo reuniões com a equipe para discussões dos casos, importante também para facilitação de uma abordagem paliativa que possua subsidios para o conforto no fim de vida do paciente.

Por fim, fazem-se importante discutir sobre a criação e aplicabilidade dos indicadores em diferentes países, considerando a necessidade de uma criteriosa avaliação devido às diferenças econômicas e culturais da população, características de cada região a fim de afirmar a importância da validação de instrumentos voltados para cada profissão ${ }^{(6,8)}$.

\section{Limitações do estudo}

Esse estudo trouxe limitações relacionadas a quantidade de estudos encontrados, nota-se uma evidente déficit de publicações relacionadas ao tema proposto por esse trabalho, considerando ainda que apesar da enfermagem entrar ativamente na realização e participação dos estudos, nenhum dos artigos apresentou foco da assistência de enfermagem em cuidados paliativos.

\section{Contribuição para a prática}

Esse estudo pode trazer contribuições para a prática baseada em evidência, por trazer um levantamento de indicadores de qualidade importantes para a assistência de enfermagem ao paciente em cuidados paliativos, visto que 
a atualização aperfeiçoamentos das práticas de cuidado é necessária. Além disso, essa revisão pode fomentar a criação de mais indicadores considerando ainda a evidente falta de publicações relacionadas ao tema e a importância de medir a qualidade da assistência a fim de identificar os indicadores positivos e negativos e assim agir para qualidade da forma de cuidado.

\section{CONCLUSÃO}

O conceito de indicadores é evidenciado pela possibilidade de quantificar a qualidade de um aspecto da assistência, podendo ainda comparar com práticas baseadas em evidências, representando assim, uma importante ferramenta usada para garantir essa melhoria na assistência. Os indicadores sensiveis ao cuidado de enfermagem voltado ao cuidado paliativo estão estreitamente relacionados com a satisfação de necessidades complexas e de vários domínios: físico, psicológico, emocional, social, espiritual e/ ou existencial. Porém, diante desse estudo pode-se observar que apesar da importância desse assunto para melhoria da assistência, foi encontrado uma pequena quantidade de referências nas bases de dados internacionais em relação aos indicadores em cuidados paliativos e nenhuma publicação voltada especificamente aos cuidados de enfermagem, porém os indicadores identificados em sua maioria podem ser aplicados nos cuidados de enfermagem

Conclui-se, portanto, a necessidade de mais estudos voltados para a área de enfermagem em cuidados paliativos sobre os indicadores de qualidade, com intuito maior de melhorar a assistência e poder ofertar um cuidado de qualidade para o paciente e família que representam o maior foco do cuidado.

\section{REFERÊNCIAS}

1. Costa RS, Santos AGB, Yarid SD, Sena ELS, Boery RNSO. Reflexões bioéticas acerca da promoção de cuidados paliativos a idosos. Saúde Em Debate. março de 2016;40:170-7.

2. Dy SM, Kiley KB, Ast K, Lupu D, Norton SA, McMillan SC, et al. Measuring what matters: top-ranked quality indicators for hospice and palliative care from the American Academy of Hospice and Palliative Medicine and Hospice and Palliative Nurses Association. J Pain Symptom Manage. abril de 2015;49(4):773-81.

3. Nakazawa Y, Kato M, Yoshida S, Miyashita M, Morita T, Kizawa Y. Population-Based Quality Indicators for Palliative Care Programs for Cancer Patients in Japan: A Delphi Study. J Pain Symptom Manage. abril de 2016:51(4):652-61.

4. Cook J, Horrocks S. Community nursing quality indicators for end-of-life care in England: identification, preparation, and coordination. Br J Community Nurs. 2016 Mar;21(3):118-23.

5. Dy SM, Herr K, Bernacki RE, Kamal AH, Walling AM, Ersek M, et al. Methodological Research Priorities in Palliative Care and Hospice Quality Measurement. J Pain Symptom Manage. 2016 Feb;51(2):155-62.

6. Lliffe S, Davies N, Manthorpe J, Crome P, Ahmedzai SH, Vernooij-Dassen M, et al. Improving palliative care in selected settings in England using quality indicators: a realist evaluation. BMC Palliative Care [Internet]. 2016 Aug 2 [cited 2019 Apr 14];15(1):69.

7. Souza MT, Silva MD, Carvalho R, Souza MT, Silva MD, Carvalho R. Integrative review: what is it? How to do it? Einstein (São Paulo) [Internet]. 2010 [cited 2019 May 1];8(1):102-6.

8. Effendy C, Vissers K, Woitha K, van Riet Paap J, Tejawinata S, Vernooij-Dassen M, et al. Face-validation of quality indicators for the organization of palliative care in hospitals in Indonesia: a contribution to quality improvement. Support Care Cancer. 2014 Dec;22(12):3301-10.

9. Martinsson L, Lundström S, Sundelöf J. Quality of end-of-life care in patients with dementia compared to patients with cancer: A population-based register study. PLoS ONE. 2018;13(7):e0201051.

10. Lautenbacher S, Sampson EL, Pähl S, Kunz M. Which Facial Descriptors Do Care Home Nurses Use to Infer Whether a Person with Dementia Is in Pain? Pain Med. 2017 Nov 1;18(11):2105-15. 